\title{
The Effect of Introversive Spatial Structure on the Behavioral and Cultural Qualities Ranging from Protection of Personal Privacy to Expansion of Social Relations
}

\author{
Fatemeh Banaei EsfaHANI ${ }^{1}$, Mohammadreza Noghsan MoHAMMADI ${ }^{2}$
}

\author{
${ }^{1}$ Master of Architecture, Islamic Azad University of Khorasgan (Isfahan), BANAEI \\ ESFAHANI (Corresponding Author), Email: banaei_es@yahoo.com \\ ${ }^{2} \mathrm{PhD}$ in Architecture, Faculty member at the Art University of Yazd, NOGHSAN \\ MOHAMMADI_Email: mohammadi@yazd.ac.ir
}

\begin{abstract}
Throughout history, the Iranian architecture and urban planning have been brimming with values and patterns that emerged and evolved over time under different circumstances. In the contemporary era, however, the transition from tradition to modernity has broken the inherent architectural values and patterns, where many of the implicit qualities have been gradually forgotten. One of such patterns in the Iranian architecture and urban planning involves introversion influenced by climatic-environmental and behavioral-functional factors. Introversion in architecture is a concept concentrating on the inner physics of the architecture, while highlighting the human intrinsic identity, where the architectural space revolves around protecting the individual privacy. Since the physical form of a space directly affects the qualities of human relationships as well as the societal culture, it is highly essential to examine the qualities of such pattern. The physical form of the space involves semantics, aesthetics and design context. So long as the form is consistent with the values and norms of its target society, it can create incentives for the development of social and neighborly relations. Hence, this study mainly attempted to identify and evaluate the implicit qualities within the introversiveive architecture and urban planning from the traditional perspective. In this regard, a comparative case study was conducted on a few samples given the qualitative aspects of human relationships, as well as the diversity of neighborly relations, especially in the context of introversion. Moreover, a historical descriptive-analytical research was carried out. Finally, there were several suggestions and solutions proposed to revive the genuine pattern of architecture and urban planning as well as the restoration of introversive architectural and urban planning in accordance with the Iranian centralist ideology.
\end{abstract}

Keywords: spatial structure, functional-behavioral qualities, introversion, personal privacy, social relations 


\section{Introduction}

As a traditional Persian and Oriental concept, introversion has long been identifying the architecture in this region. In fact, it is one of the most prominent features of Iranian architecture and urban planning underlaid by the public and local ideological systems. The philosophical sense of introversion in the Iranian traditional architecture originates from the human return to the self and the centralist ideology. Concentration on the protection of personal privacy is the most important characteristic involved in the introversive style. In particular, this architectural principle is crystallized in the physical organizing of a house as the most private space of human life and the first and foremost reflection of identity at the individual and societal levels. In addition, the climatic and security factors have been contributing to the development of introversive architecture.

With the passage of time and the spread of globalization, especially from the beginning of the Qajar and Pahlavi eras, the Iranian approach to introversion evolved. The needs and desires of the modern human drifted apart from what the ancient people conceived, giving a new sense to introversion. This study intended to examine the introversion institutionalization within the traditional architecture and urban planning in the historical context of Yazd as the best example of Iranian traditional architecture. In this regard, they study explored the emergence of extroversion due to the modern effects into to the contemporary era. Then, a comparative study focused on the content dimensions of traditional introversion against the modern version, thereby to assess whether it can be employed as the Iranian rich architectural content. Finally, several strategies and suggestions were put forth.

The one question arising in this respect is why there is a major concentration on introversion and assessment, and whether it can be employed within the modern architecture and urban planning? In response to this question, bearing in mind that the tendency to individuality in today's life is one of the biggest problems of social relations at neighborhood and family spheres, it is crucial to build the desire and motivation to enhance social interactions under theoretical discussions of behavioral science and human relations. This issue requires several factors to be examined, but the most important factor involved in architecture is the spatial structure playing a key role in enhancing or weakening social relations. The role of physical factors and human parameters in creating a sense of belonging to the environment is paramount. The physical form of space covers semantic, aesthetic and contextual dimensions, which can be influential in motivating the formation of neighborly relationships as long as they are consistent with the values of the society. Particularly in more private realm involving the neighborhood of individuals, it can be crucial as a small community where people live in a complex.

By comparing the relationships in old and new neighborhoods, it can be concluded that urbanization (living in apartments) and elimination of publicly shares spaces in recent decades have curtailed the social interactions. In the past, good neighborly relations were built based on the horizontal development of neighborhoods and the open central space. Nowadays, the neighborhoods have developed in the vertical direction, where there are no central courtyards, 
opening gates and vashodgahs ${ }^{1}$. This has led to lower tendency toward building social relations between neighbors. This is a general overview of the early stages in the desk review of relevant literature. During the field and comparative studies on two types of old and new textures in Yazd, it was found that the design of urban spaces and neighborhoods based on introversive spatial structure in its modern definition can significantly improve the quality of social relationships. The sense of intimacy between neighbors can be enhanced by taking advantage of functional qualities in spatial elements such as central open spaces, squares and vashodgahs in neighborhoods and central courtyards with respect to personal privacy as a bridge (interface) between the public and private arenas, which in turn can curtail the adverse effect of vertical urbanism on social relations. Moreover, the neighbors can interact with each other safely and effectively in compliance with human dimensions within the neighborhood and providing the conditions for engagement of various social groups.

In relation to the necessity and importance of this study, it should be noted that poor identity the architecture of houses and residential areas today, loss of living standards, fading personal privacy and many hierarchies in the modern architecture made it inevitable to address the issue. An additional aspect of this study was the possibility of gaining more insight into the rich architecture in the past and restoring the architectural patterns of today can enhance the quality of current residential spaces and promoting the concept of privacy to individual and family lives. Given the originality embodied in popular culture of the Iranian society and qualities arising from introversion within the Iranian architecture, including the architecture of traditional houses in Yazd, it seems such patterns can be identified and leveraged. These patterns can be used to design new neighborhood residential complexes for identification and optimization of the concept of introversion and revival of privacy.

\section{Overview of the characteristics in neighborhood complexes from the traditional eras}

\subsection{Structure of traditional neighborhoods}

In the past, urban neighborhoods were developed and named based on different social classes, businesses and followers of different religions. This social and cultural solidarity led to psychological belonging to the community and adherence to unified customs as well as a sense of differentiation compared to other neighborhoods [1].

Some of the old houses in Yazd were built and combined together for family reasons, creating a valuable collection. The familial complex of Arabs in Fahadan neighborhood is an example where there are several sub-passages connecting to a home, providing access to other passages within the neighborhood leading up to the market in the city center. On their way, the main passages cross the neighborhood centers which are actually located on the main routes.

1- A vashodgah was a local open space within the traditional structure of the city, where the spaces and gathering places were divided in the neighborhood. 
Natural elements such as water, earth, sun, wind and plants are essential in the foundation of traditional architecture. That is because the traditional architecture is based on climate and geographical environment. These principles include:

- Respect for human introversion and return to self

- People-orientedness and concentration on the human scale

- Avoid any frivolity, unnecessary glory and decoration

- Effort for self-sufficiency and utilizing the aborigine resources and materials

- Refraining from any dependence on foreign agents

- The application of appropriate measurement units for structural proportions in terms of accessibility and appropriateness of design and beauty

- Avoiding any large-scale design leading to materials waste and excessive costs, not to mention the unreliability of structural strength [2].

In traditional neighborhoods, the spatial order of elements is organized around the core neighborhood. One of the most identifying elements associated with texture is the hierarchy of access points, which varied from one arena to another, progressing from public into semipublic and then into semi-private and private arenas. The urban structure has developed inwardly from the market to the passage, passage to opening gate, hashti, corridor to the niche and then courtyard. The same hierarchy has been observed in the domestic sphere of houses. Thus, the residential context combines with houses as the smallest and most private elements of the texture within the neighborhood as a set of repeating elements with a specific function, shape, size and proportional volume [3].

\subsection{Social relations prevailing in the residential quarters in the past}

In the past, there were several factors contributing to the human communities such as geography, climate, defense, religion, culture and economy. Based on these spatial factors, there were complexes formed with certain physical and social boundaries. Urban environments served to satisfy the social needs while houses met the need to families. Urban spaces where citizens communicated and the people gathered at community centers according to ancient traditions, celebrations, festivals and religious ceremonies. Thus, urban spaces in the past entailed a limited realm characterized by specific aesthetic and functional qualities, setting the ground for purposeful engagement of people in the community, greatly contributing to the living standards and the public culture.

In the past, the urban texture was almost identical and family members living in one neighborhood with a sense of attachment based on kinship and marriage. From birth to marriage, childbearing and marriage of children, family members lived in one neighborhood. In the contemporary era, as the lives of traditional Iranian family has evolved away from the traditional style, thus altering the function of urban neighborhoods. Families separated from their old neighborhood and settled in a neighborhood given their financial situation and the evolution of culture had to build the new relationship similar to the kinship back in their old neighborhood, thus developing the need to "neighborly communities". Over time, however, such need turned into social partnership as the introversive spatial structure evolved into 
extroversive approach to a new desire for individualism and diminishing neighborly relationships.

\subsection{Familial communities}

The physical structure of a residential complex in the past was based primarily on the cultural and social characteristics of their residents as well as the economic conditions and regional climate. As for the familial communities, however, there were social forces proving stronger than other factors. The families in Yazd are characterized by their unique cultural and social characters such as hospitality, family, friendship, inwardness, religiosity, need for privacy, security and simple lifestyle [2].

In the past, families formed in such a way that members of an ethnicity inhabited together while each protected its privacy observed through various courtyards. The social relations between the residents of these communities and their neighbors maintained the privacy and security as a physical hierarchy of opening gates, hashtis, corridors and courtyards, which provided social interaction between the residents. The opening gates connected the neighborhood units, providing a desirable sport for neighborly communication. The identify was each neighborhood was outlined by several indicators such as wind towers. The luxuries were minimal, since it eliminated the gap between the poor and wealthy classes of society. Between social classes, rich and poor to raise their minimum. The buildings and the courtyards were designed in direction of Qiblah. Moreover, tall walls were constructed in family homes to create an introversive humble structure, while protecting the privacy of houses.

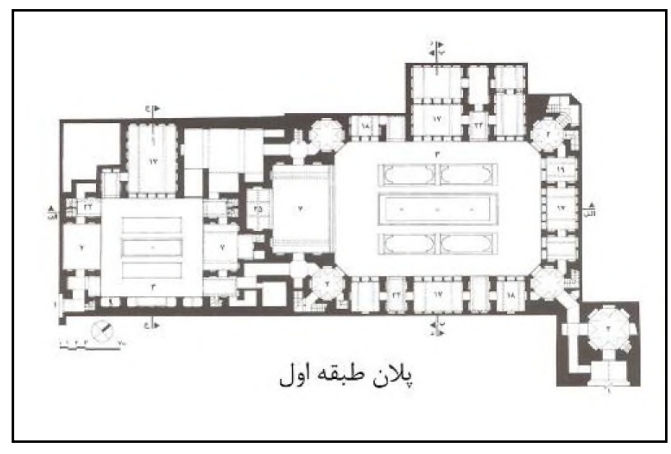

Figure 1: First floor plan of Mortaz House

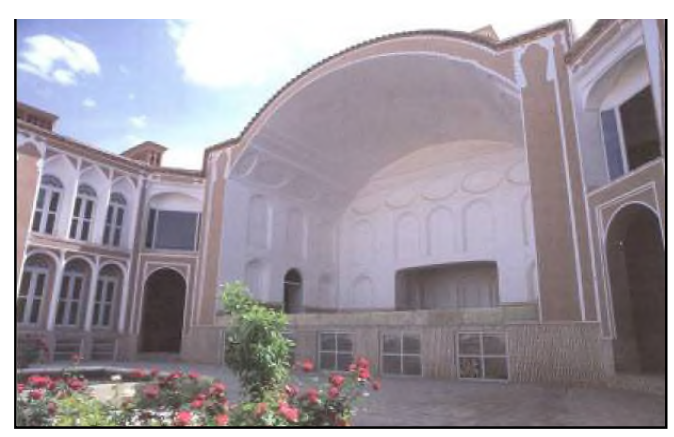

Figure 3: A view of Mortaz House

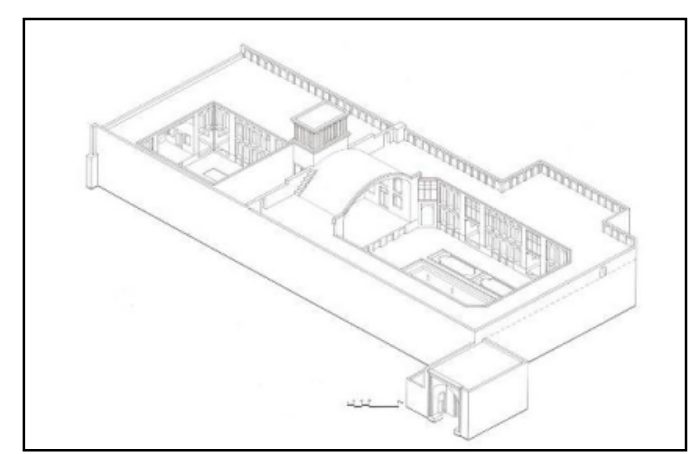

Figure 2: Three-dimensional view of Mortaz House

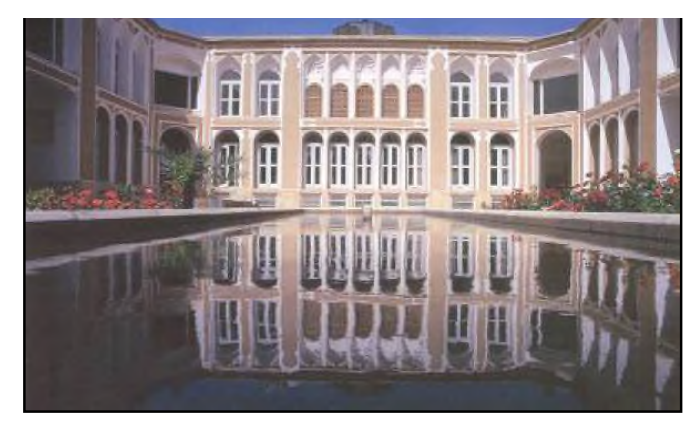

Figure 4: Another view of Mortaz House 
Thus, the social and cultural characteristics along with climate and economic factors play an important role in improving the quality of neighborhood formation. The outcome is a set of neighborhood communities where there is tranquility, comfort, a sense of belonging, intimacy, solidarity and religious belief established in people's lives.

\section{Contributing factors to the formation of introversive architecture}

As mentioned in previous discussions, the main factors contributing to the introversive architecture is primarily climate and then environment, behavior and function, according to which essential measures should be taken to provide favorable living standards.

\subsection{Relationship between introversive structure and climate}

In the past, the buildings, especially in architecture of houses, were constructed based on the environmental conditions and compliance with the climate. In hot and dry climate, the buildings took a closed shape with tall walls covering the open intermediate space (central courtyard) entailing minimal corners and bends as the basic feature of Iranian traditional architecture [4]. Moreover, the spatial hierarchy facilitated the exchange of heat between the home and outdoors. Embedded elements such as hashti, wind deflector, pergolas, sash window, tabeshband, porch and niches were crucial in conceptual and cultural terms. Hence, the ancient architects designed the building in such a way to achieve maximum correspondence with its own regional climate as well as maximum comfort for its residents.

\subsection{Relationship between introversive structure and behavior}

The quality and mechanism of activities within urban spaces depend on cultural factors and environmental factors (physical space). According to cultural factors including customs, habits, traditions and ways of life prevailing in each geography, the human outlines a specific individual and social relationship with space and its interactive elements.

In environmental psychology, behavior is explored in interaction with the physical, architectural and symbolic dimensions. Humans are affected by environmental conditions on the one hand and organize them according to the needs, values and goals of their environment on the other hand. There is a mutual relationship between the human impact on the environment and environmental impact on humans. Given that the individual behavior within a space directly depends on the physical structure designed for that space, the surrounding environment must be designed in such a way that can provide the optimal functionalities. Moreover, the qualitative aspects of the interaction between behavior and environment can be assessed through the question whether "loneliness" is better than "togetherness" from the human point of view. In this regard, Irwin Altman employed the results of other scientists, presenting four key concepts for understanding social interactions of people in different cultures including "privacy, personal space, territory and crowding" He introduced privacy as the core to linking the concepts, arguing that privacy is an important contracting process where the individual or group connects others; personal space and territory involve 
mechanisms to achieve the desired level of privacy; And finally, crowding is a social situation due to the absence of efficient mechanisms of privacy leading adverse social relationships [5].

In relation to the circle of human needs for privacy and society, it can be argued that human will achieve the growth, excellence and crystallization of capabilities through both public and private lifestyles, which cannot be separated. The need for private life in the family level as the natural need is as important as the need for social relations and facilitating the promotion of human societies. An example is family communication with relatives, neighbors, the community, etc. Meanwhile, "neighborhood" is a form of social and cultural physical realm significant in response to this need in the Iranian urban structure. Although this concept has evolved through changing conditions of human life in economic, cultural, social terms and development of technology, it is still inevitable to be explored and discussed.

In the geographical scope of this study, the relationship between the introversive structure and behavior derived mainly from the local people from concepts such as privacy, personal space and introversion, affecting the behavior and function. This concept can be explored in terms of social life and relationships in the neighborhood and in the more private relationships (familial relationships) in the past and current periods.

\section{Transition from private homes to neighborhood complexes}

As the most private space of human life, a house fulfills the human natural needs, including the need for security, attachment, a sense of belonging, peace, stability etc. The human being experiences a sense of belonging and finds identity in the safe space of a house. What was called a house in the past, however, is distinct from the modern apartments. Traditional house originates from a vernacular architecture in the context of each regional entity which is formed mainly in the period before the modern era. It takes in the unique local architectural features, achieve a specific, coherent and deep-rooted identity within the traditions of architecture.

People in the past respected the realm of humans and privacy. By building high walls and introversive structures, they fulfilled the climatic requirements while protecting the house from the intruders. The spatial hierarchy was yet another feature of introversive houses meeting the climate and security measures and the protection of personal privacy. Thanks to the abundance of cheap land and energy resources in the past, open spaces were expanded horizontally in one or two floors. The set of house architectural elements entailed a completely personal privacy, since the architectural elements were embedded around the central courtyard, forming a closed endogenous volume. The spatial structure sequentially developed from the most private spaces like chambers and bedroom in the back of the house all the way to the central courtyard, corridor, hashti and opening gates. Hence, a compliance with the spatial hierarchy led to the division of spheres. The neighborly communication was established through opening gates between several personal homes. It functioned as a shared passage but nearly private (semi-private arena) exclusive to the residents, leading to the side streets and alleys of the neighborhood. That was how privacy and personal space were concerned in the past. 
With the expansion of globalization from the beginning of Qajar and Pahlavi eras, the introversive architecture gave way to the western extroversive architectural style. The consequence of such developments could be found in behavioral patterns altered due to mismatch between the new structure and the climate. Nowadays, there are many factors changing the household patterns such as urbanization, high prices and scarcity of land and materials, excessive costs of energy, cultural shift and changing human needs and desires as a result of globalization. Hence, vertical houses provided smaller dimensions and more extroverted physical dimensions, while eliminating many traditional functions. Finally, the physical structure and the value of the introversive house yesterday turned into residential neighborhoods and apartments. As architectural structure altered the qualities of neighborly relations, the vertical extroversive structure curtailed the personal privacy, where the public and private arenas lost their link. Thus, the issue led to lower tendency toward social contacts in the context of neighborly relations. This is where the importance of studying the interaction and behavior of physical space becomes evident.

Today, people have adapted to the condition of living in apartments. However, in order to promote a sense of inner satisfaction, calm and satisfaction of the needs and demands of personal and social desires, it is crucial to explore the traditional introversive architecture in terms of physical structure, values and symbols so as to preserve it according to the conditions of human life today. For instance, it is critical to examine the climatic conditions, respect the privacy and personal space, comply with the hierarchy in the formation of architectural arenas in proportion to the introversive pattern as elaborated on in this section.

\section{Features of modern neighborhood spaces}

With the advent of modernity in the cultural, social and economic system, the urban communities transformed. By the involvement of machines in the lives of people, the physical shape of alleys and passageways turned from organic into large-scale intersections and squares. Following the rural to urban migration, the indigenous lifestyle altered by the western patterns, leading to different public demands and needs. On the other hand, the conversion of bazaars into huge commercial markets, zoning policy and land use in urban renovation projects separated the living and work spaces.

Hence, what was consistent with the traditional Iranian life was altered within the urban social system. The consequence of this transformation in the social life and the neighborly relations was the short-term rigid relationships as opposed to the past. The past human spaces lost their credibility. Cross streets and squares no longer reflect the characteristics of urban spaces, and there are no attractive elements for the public. The modern routes only facilitate the traffic of vehicles. The presence of vehicles on the road network, people can no longer communicate directly with spaces since the interconnections have faded leading to further social isolation. In the modern city, houses have been arranged in a row and next to each other lacking the introversive physical structure. Instead, the introversive and extroversive styles have been blended in the modern houses without paying attention to the privacy in the design. 
In general, the adverse factors that jeopardize the modern residential texture include peace disturbing (noise pollution, air pollution, insecurity), comfort disruptive (negligence of climate, dependence on city center), sense of place (lack of vitality, fading social solidarity, weak sense of cooperation and collaboration, scale, independence from the environment), fading influence of religion (weakening of the Mosques in the residential complexes, removal of privacy and hierarchy of vision (extroversion) and the destruction of private and semiprivate spaces, unfriendliness of spaces (poor response to the needs), classification of housing complexes on the basis of location, recreational facilities, price of land, buildings and public economy, breakdown of complexes into the slums and affluent sectors, disturbance (conflict and alienation between the elements), lack of dignity and identity [6].

\section{Emergence of extroversion in contemporary houses}

Concurrent with the Pahlavi dynasty and promotion of modernism in Iran, the family-social structure evolved. Nuclear families expanded and consequently changed the form of residential buildings. For the first time, the corporate residential units and new urban areas formed, intensifying the tendency toward independent and modernized houses. As a result, the old textures in streets Imam, Salman, Kashani, Iranshahr, Farokhi and Taleghani turned into new residential functions. Moreover, the interconnected houses were constructed by the government across Maskan, Shahrsazi and Iranshahr streets. In fact, a new texture developed in this period known as intermediate texture. It was in fact formed from 1921 to 1961. However, this was contrary to modern street development within the old texture. That was how the city transformed and construction of new streets in the central area disrupted the older texture [7].

Gradually, the traditional style of houses with a central courtyard turned into extroverted houses opposite to the traditional introversion in terms of physical structure, which has been illustrated in figure (1). The quality of housing changed, and elements such as porch and yard were eliminated and replaced by balconies. Unlike the past, the new mass of structure developed vertically downgrading the quality of neighborly connections.

\section{Overview of the reasons behind transition from introversion toward extroversion}

In order to investigate the causes behind extroversion as an alternative approach to introversion, it should be stated that such trend can be traced back to the evolution of human thoughts crystallized objectively in the architecture. Since then, the modern man faced different needs and desires concerning a new house given the intellectual developments and changing behavioral patterns.

In addition to these fact, housing as one of the basic needs of human beings has become an economic problem due to migration and population growth in large cities today. This issues were tackled by a policy adopted after the Islamic Revolution known as Clean Project 
(standing for three concepts of savings, mass housing, downsizing in Persian) enforced within the second and third Five-Year Development Plans [8].

In addition to the foregoing, there are other factors changing the patterns of housing such as

- $\quad$ providing energy sources required per household in housing units with large areas

- $\quad$ Prevention from land waste

- Considering the financial capacity of the majority of people for affording a house

- Helping to save on the use of public funds allocated to housing in the country

- Achieving the best urban dense model and prevention from uncontrolled urban expansion

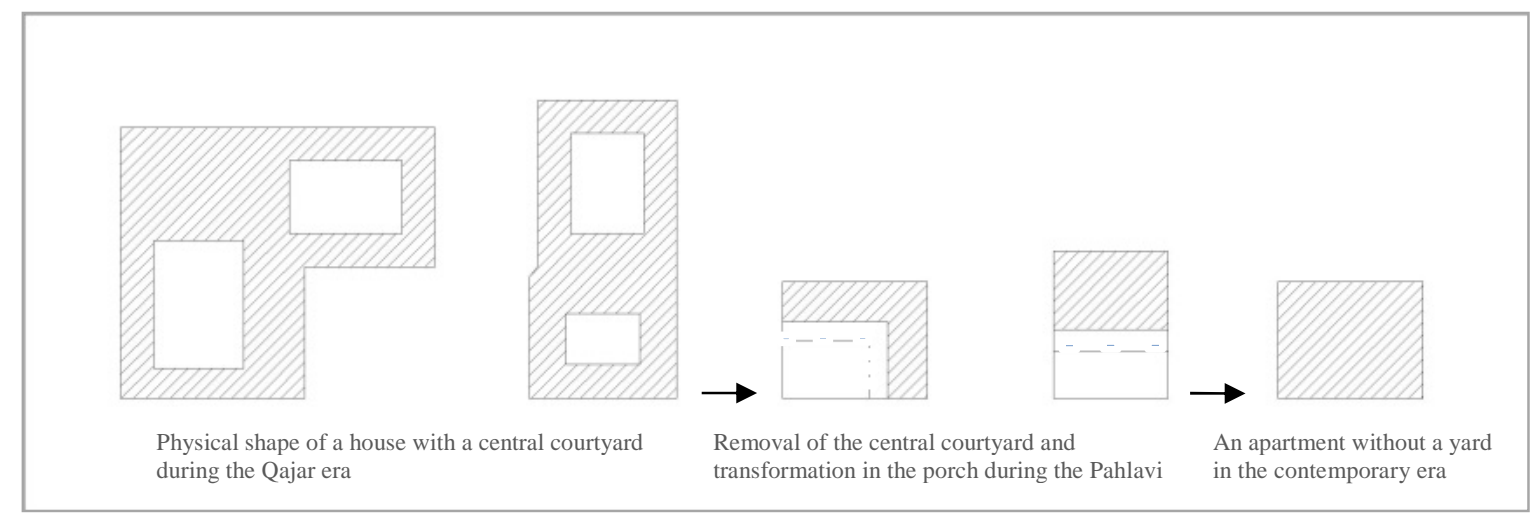

Figure 5: Changes in the physical shape of the house and the elimination of porch and yard from the Qajar era to the contemporary era

The effects of modernity in the transition from introversion to extroversion in Yazd as origins of the genuine traditional pattern led to certain changes in the housing evident these days. Then, the concept of introversion was modified from the human perspective as the traditional pattern of introversion was gradually forgotten. Moreover, given the inevitability of changes resulting from the passage of time, and factors such as economic problems in housing, difficulty of energy supply due to the growing population and changing patterns of human behavior and changes in the needs and demands of humans today, a new introversive pattern can be adopted as a golden rule to design different houses fulfilling the human needs from the introversive point of view.

Despite the physical organization of houses such as reducing the size of houses and the expansion of the floors, many of the qualities of traditionally introversive architecture can be taken as fundamental principles. For instance, an introversive house can be designed in the modern sense by maximum use of natural energy, creating the conditions for equitable and appropriate communication between neighbors, according to mutual rights, sense of belonging and social solidarity and a new identity given by cultural, recreational and sports activities. Apart from adoption of genuine old patterns, such design will grant a unique identity to the modern houses, while taking into account the climatic, socio-cultural, religious and symbolic aspects especially in Yazd. Such novel patterns can be devised not only to fulfill 
what today's man craves for, but also to establish a firm bond between tradition and the modern world.

\section{Reflections on the physical-spatial qualities of several neighborhood complexes according to the historical texture of Yazd}

There are a few examples of contemporary neighborhood complexes where the spatial structure has been adapted from the traditional texture of Yazd, including: The Ilchikhani residential complex, Tal neighborhood residential complex and Shahriari Godal residential complexes. These neighborhood complexes entail high quality spatial patterns influencing of the development of urban design ideas, the promotion of behavioral patterns and tendency toward social participation.

In the few samples under study, effort was made to design a house within the historical, life and vitality contexts. The strengths of this complex were proximity to urban centers and services, reduced costs, the use of spatial perceptual qualities. In these residential complexes, the social relations of neighbors can be improved by define the semi-private arenas such as entrance, foyer and common courtyard. Green space and avoidance of closeness can leave a positive impact on the quality of neighborhood intuitive and visual qualities.

Such models of housing are more desirable for building neighborly relationships owing to the spatial and physical qualities creating a sense of belonging and close relationship between the individuals as well as the type of organization and hierarchy of spaces and arenas and also
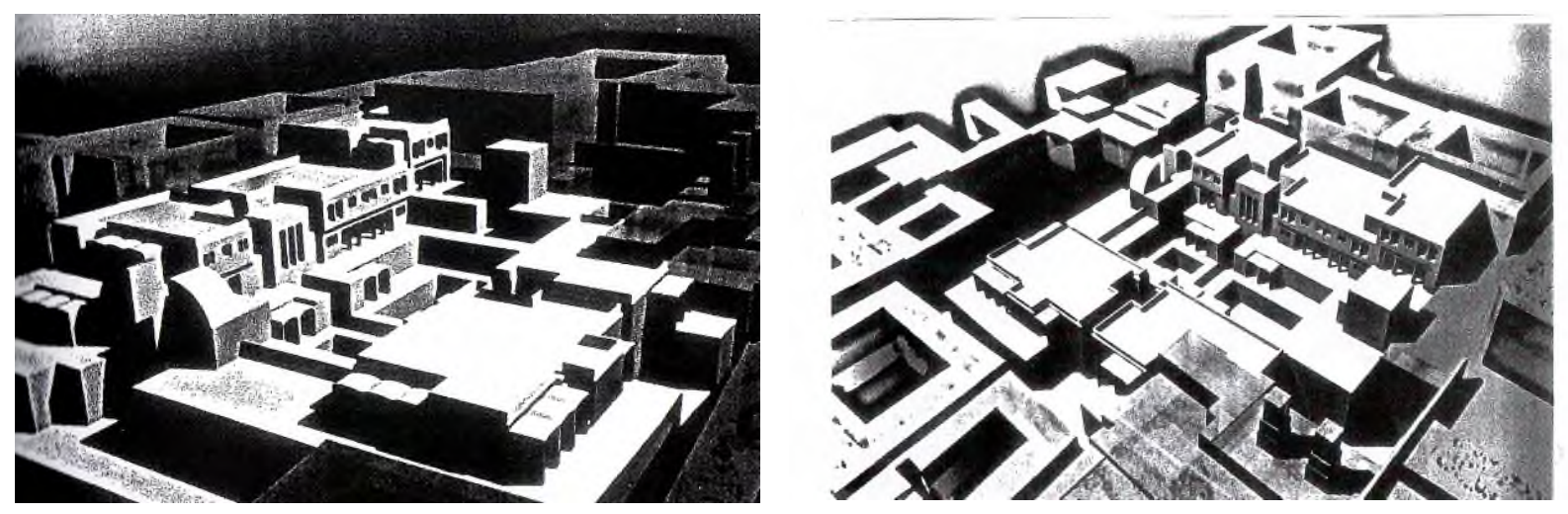

Figures 6 and 7: View of a residential neighborhood known as Ilchikhani in Yazd

Some features of this neighborhood complex have been briefly listed below:

- Factors contributing to the formation of neighborhood units can be organized social, cultural, security and climatic issues (somehow in retrospect).

- Factors contributing to the creation of privacy and separation areas (from semi-private to public) within the neighborhood are religious and cultural features such as privacy, social (relationship neighborhood relationships-kinships), psychological, comfort, etc. 
- Techniques employed in arrangement of arenas: Mazing in the neighborhood plan, adding light and dark, framing the routes with tavizeh and sabat, shifting in proportions, staircase and shifting in the walls, etc.

- Reasons for using green space in the common neighborhood area is to reduce private open space and create the right conditions in the space for engagement of the neighbors.

- Embedding an entrance for the neighborhood complex by creating sabats, tavizeh, changing proportions and breaking the field of view.

- Creating different spatial qualities in the neighborhood space through open air tunneling, sabats, tavizeh, sunshade, staircase, wide and narrow passages.

- Establishing order and diversity together leading the desirability of open spaces.

- Unity drawn from the spaces and elements are rooted in the same principles of design, construction and structure. Therefore, any amount of difference and diversity in physicalspatial manifestation of this principle given the common rules and regulations would not give rise to confusion and disorder.

- By creating shade in many hours of the day, the streets and public open spaces managed to balance out the warmer months of the year during the unfavorable climatic conditions (extreme temperature and sunlight).

- Density and compaction of grains is the main factor holding favorable conditions and cold temperatures in the hot summer months at large scale, texture and the neighboring unit.

- The rigid materials and non-transparent outer walls of the houses provide favorable climatic conditions and lower exchange of inside and outside.

- Creating a microclimate and covering the open spaces made by the masses can provide ideal climatic conditions inside the building with air-conditioned.

- In order to induce a sense of identity, indicative elements such as windcatcher, pergolas, awning, sabat and central courtyard can be designed in the complex.

- The homes and textures should not be connected only directly through the entrance, since it minimizes the communication between the residents and the complex, thus creating a sense of insecurity and non-intimacy.

- On the streets and sidewalks, diverse elements should be used to convince people to stay in the space and handle the environmental elements.

- In the spatial organization, organic checkered network can simultaneously create diversity and avoid uniformity.

- The full and empty spaces should be such that filled spaces cover the empty spaces, where greater sense of security and identity is involved in addition to providing climate.

- Application of elements such as tavizeh and sabat along the passages observes the climate issues, while creating human proportions, according to aesthetic aspect of rhythm and highlights.

- Attention should be paid to the issue of privacy and field of view to other houses, the streets and complexes. 
- Observing the spatial hierarchy and shifting of arenas from the most public to the most private spaces.

- Application of deadend alleys between mass and space can fulfill the climate issues and spatial arenas in addition to the sense of safety and belonging to residents of the neighborhood.

- Embedding playgrounds for children in the vicinity of green spaces for meeting the needs and demands.

- In terms of visual impact and quality, the coordinated skyline of the complex and the surrounding texture should be considered.

- Property boundaries must be set correctly, so that a few buildings are separated by a certain space.

- The complex design should account for maximum consistency between the volume, height and surrounding texture.

- To avoid the annoying winds and intense sunlight, windcatcher and sunshade can be used. Moreover, the use of canopies, wind barriers, planting trees and other suitable plants along the sidewalk can encounter the troublesome winds and intense sunlight.

- To take advantage of the favorable environment, the courtyard and green space should host several benches and places in the direction of favorable winds and breathy trails in summer or winter sunlight. The use of deciduous trees provides the chance to sit in the shade or sun at different times of day.

- Creating indoor spaces like porches and pergolas that will create a shadow or a few tents in the gardens and yards.

- Creating masses built around open spaces away from the open spaces can provide a microclimate where embedding green space and waterfront can lead to air conditioning, while enclosing the spaces against climatic factors.

- Using indigenous architectural elements such as sabat, tawizeh, narrow alleys and high walls can provide shade and protection against the wind and the storm.

- The longitudinal direction of the complex toward Qiblah (south and 45 degrees east) can provide maximum amount of shade from sunlight exposure during the hottest days of the year allowing maximum depth of light into the room on the coldest days of the year.

- Appropriate arrangement of skylines and ground alignment meets the architectural aesthetics while providing windcatcher in the higher spots against the disturbing winds. As the wind direction shifts, it lowers and ends at a height of one storey.

- Setting and balancing the combination of mass, space, density and floors

- Linking the public and private spaces by serial connection for provide flexible spaces

- Compliance with the principles governing the spatial qualities such as order, unity, diversity, spatial sequence and closeness

- Striking a balance between built and open spaces in the residential complex

- Creating visual and motor axes and rotation axes coordinated and harmonic to its hierarchy

- Concentration on the spatial organization to combine mass and space (radial, series or combined arrangements)

- Creating diversify in the skyline as well as taking advantage of the maximum natural wind and sun energy and employing the staircase patterns, spiral etc.

- Minimizing the blind and out of sight spots leading to potential offense. 
- Using bright colors in facades

- Designing the neighborhood complexes so that the diversity necessary for life experiences is provided at different levels. Living in open, semi-open, closed and indoors spaces (using the roof, terrace, courtyard)

- Making an altitude difference for using the roof with high-quality materials creating better conditions for residents as well as shading by objects higher than the roof level.

- Creating diversity in architectural space by shifting the scale and scope, changes in the type of spaces, closeness level, shades, sidewalk space and green spaces, outdoor and indoor spaces, mass and space

- Using the vaulted form in the aesthetically attractive view (observing the diversity and proportions in the facade) as well as to recourse to the traditional architecture combined with modern architecture

- Embedding the opening in combinational arrangement in the walls toward the street given the changing human attitudes towards introversion and enrichment of people with different tastes

- Covering open spaces with pergolas and trees as Garden City, which makes a microclimate offsetting some of low moisture.

- Employing a domed form on the roof to reduce sunlight, provide light exposure from the roof and utilize the aesthetic aspects of its form

- Creating a compact and shadow-filled texture at high altitude compared to the width of the passages

- Considering a great deal of height for windows according to climatic conditions (reflected rays from the ground) while observing the privacy of houses

- Separating the roads and access routes for people on foot and driving

- Striking a balance between private and communal property: Since the communal spaces including a central courtyard and private yard are shared between the neighbors, they can provide a cozy atmosphere for families while enhancing the social interactions and meeting the neighbors. At the same time, creating privately owned spaces for each household is required to comply with the spatial hierarchy.

Considering these factors in the introversive neighborhood complex can maintain personal privacy in addition to the principle of confidentiality, leading to an increased willingness to neighboring relationships, greater diversify and attracting people to the living environment. Such environment can provide the most favorable living conditions for people based on respect for religious and cultural beliefs and increasing the sense of belonging and attachment to the place.

\section{Association of urban texture the neighborhood complexes}

A desirable neighborhood complex should establish good relationship with its surrounding texture. Especially in the design context, it becomes more essential within the traditional texture adjacent to and high-value historical elements.

In this regard, there are several facts as follows: 
- Space conjunction with the neighborhood complexes and their surrounding appearance (coordinating objects, surfaces, lines like edges and skyline, paints and building materials with peripheral elements)

- Harmony of spaces and architectural elements of complexes with the surrounding textures

- Functional and behavioral coordination between the complexes and the environment, such as access points and pathways, spaces of crowding and squares

- Compliance with the hierarchy in harmony with the surrounding texture, such as neighborhood, street, street and city

- Striking a balance between built spaces and open spaces

- Coordination and consistency with the lifestyles of residents and the surrounding texture

\section{Conclusions:}

In designing of residential complexes, it is not only about construction of individuals physical structures, but it involves an exploration of factors such as the environment and the neighboring qualities. A complex is required to fulfill the physical, cultural, social and functional needs of the residents. Since direct modeling of the historical context along is not able to respond to the demands of this study, the concepts were redefined under quantitative and qualitative aspects (physical and functional). Therefore, several points can be summarized as follows:

1- In designing a neighborhood complex, it is crucial to connect the neighborhoods units together as a whole as well as communication with the surrounding texture. Hence, the factors contributing to the quality of the built environment should be considered in the design.

2- The connection between the built spaces can be realized through open spaces and providing functions such as green space, seating areas and children's playground, which create a sense of belonging and willingness to stay in the living space based on the quality of architectural space.

3- The Bio Complexes should meet the demands and needs of cultural, social, customary and appropriate to the character and mentality of the inhabitants.

4- Spaces that should be designed in a desirable neighborhood include: Private living space owned by the individual and the family atmosphere at the micro level by creating areas of private, semi-private, public and semi-public, semi-private space belonging to neighbors and semi-public spaces belonging to a neighborhood aimed at creating social interactions with their surroundings and meeting the needs of the social communication

5- An appropriate design focuses on social stratification of the families in the complex. Moreover, a good understanding of the beliefs of people or family members can reveal many of the issues in everyday lifestyles.

6- The complex elements should entail high-quality physical and spatial properties so that the individuals can gain greater sense of attachment.

7- The neighborhood complex should be designed proportionate to the masses as well as the type of neighborly communication, circle of familiarity and intimacy. 
8- A case study on economic conditions, culture and climate of each region and the behavior of various social groups, genders and ages cab identify the needs and desires, which in turn can yield cost-effective construction projects for neighborhood complexes.

9- Case studies on behavioral characteristics of residents serve to determine the form and size of neighborhood household and outline the relationships between the residents.

\section{References:}

1. Haji Jafari, Sh. (2007), "designing a neighborhood unit with an emphasis on social neighborly relationships", Master's Thesis, School of Architecture, University of Yazd

2. Pirnia, M. (1999), "Introduction to Islamic Architecture", Editor: Memarian Gholam Hossein, Tehran, Iran University of Science and Technology, Fourth Edition

3. Banaei Esfahani, F. (2011), "Darolshafa restoration project in Yazd" Cultural Heritage and Tourism Organization, Yazd

4. Memarian, Gh. (1994), "Introduction to Persian residential architecture; introversive typology", Iran University of Science and Technology, Tehran

5. Altman, E. (2003), "Environment and social behavior: Privacy, personal space, territory and crowding“", translated by A. Namazian, Tehran: Shahid Beheshti University Press. First edition, P: 5

6. Ozinejad, Massoudeh. (2002), "Khoramshah; past symbol, future presence (designing a residential complex in the neighborhood of Khoramshah)", Thesis Master of Architecture, University of Science and Technology, Tehran

7. Alavi Kia, A. (1995), "Analysis of the spatial structure of Yazd”. Azad University of Najaf Abad, Esfahan

8. Armanshahr Engineering Consultant Company, (2007), "Strategic Plan for wornout texture of Yazd," Housing and Urban Development Organization of Yazd, Tehran-Yazd 